\title{
Investigation and Analysis of Mental and Behavior Symptoms of Alzheimer's Disease Patients
}

\author{
Zhenghai Sun, Huimin Han, Fengwu Yan, Jianzhong Li, Wenlin Wang, Yunfeng Han, Jingwen
} Meng and Na Wang

Fund Project: Heilongjiang Province Health Department Project Qiqihar City, Heilongjiang Province, senile dementia epidemiological study (2012-344)

Corresponding author: Sun Zhenghai, Qiqihar Medical University, Qiqihar 161006, China E-mail:zhsun1980@sina.com, Tel: 13763456007

Corresponding Author: Sunzheng Hai (1980-), male (Han), Qiqihar,The lecturer of mental health college in Qiqihar medical University, MA, In 2014 as a visiting scholar at Peking University psychiatric professional training to learn, is mainly engaged in research science aspects of Geriatric Psychiatry. E-mail:zhsun1980@sina.com, Tel: 13763456007

Objective: To assess the mental behavior of patients with senile dementia. Methods: A stratified multistage cluster sampling method was used to identify the subjects. The screening and clinical diagnosis was performed. The screening phase was carried out by the Simple Mental State Scale (MMSE) and the Daily Living Ability Scale (ADL) test. The clinical diagnostic phase was assessed on the basis of history, clinical physical examination, and neuropsychological tests, using DSM-IV and NINCDS-ADRDA criteria. Neuropsychiatric questionnaires (NPI) were used to evaluate the mental behavior symptoms of AD patients in patients diagnosed with AD. Results: (1) A total of $83.91 \%$ of patients with senile dementia experienced varying degrees of mental and behavioral symptoms in the past month. The most common symptoms were anxiety (54.02\%) and sleep / nocturnal behavior (52.87\%). 4.60\%) and emotional high / euphoric (5.75\%), the other mental behavior symptoms have a higher incidence. (2) This study shows that awareness of dementia and attendance rate (less than 5\%) are relatively low. Conclusion: (1) The incidence of mental and behavioral symptoms in patients with senile dementia (AD) should be high attention (2) The awareness rate and attendance rate of senile dementia (AD) should be improved, and early intervention of prevention and disease should be done The

Keywords: senile dementia; psychosomatic symptoms; neuropsychiatric questionnaires

Behavioral and psychological symptoms of dementia (BPSD) are dementia, sensory, mental, or behavioral disorders in dementia patients. $\mathrm{AD}$ patients in the progressive cognitive decline at the same time, often accompanied by loud noisy, too much activity, but also accompanied by delusions, paranoia and so on. In 1996, the International Society of Geriatric Psychiatry held an international consultation to define the mental disorders or behavioral disorders of dementia, which were referred to as BPSD. BPSD is far more than its cognitive symptoms should cause people's attention. About $70 \%$ to $90 \%$ of dementia patients in its course of a certain stage will appear this symptom [1]. BPSD not only causes dementia patients to their pain and loss of function, increased the burden on families and society, especially to care for those who bring great difficulties, but also the type of hospital admission and hospital care important reason. Non-cognitive aspects of Alzheimer's disease should be of concern and concern.

\section{Materials and methods}

\subsection{Research methods and objects}

The survey was conducted in a two-stage approach (screening and clinical diagnosis) in line with 
international standards. The investigation team consists of neurologists, neuropsychological and psychiatrists and senior medical students with relevant knowledge and with some clinical skills. In the screening phase, a unified questionnaire and a standardized survey were used to carry out the questionnaire survey, and the cognitive function test was carried out with the Chinese version of Simplified Chinese Mental State Scale (MMSE). Serious cognitive impairment or aphasia and other reasons, cannot be MMSE scale assessment, insiders believe that there are cognitive impairment, can be judged according to clinical manifestations and ADL score. MMSE abnormal decision to adopt cultural adjustment of the demarcation value, illiterate $\leqslant 19$ points, primary school group $\leqslant 22$ points, junior high school $\leqslant 26$ points. Clinical diagnostic samples included those who screened positive in the first stage, and although MMSE points in the normal range, the screening staff or family members suspected significant cognitive impairment in the elderly and the MMSE score was scored at a certain percentage. At the same time mental psychological tests and behavior assessment, including the daily living ability scale (ADL), Hamilton Depression Scale (HAMD), Ischemic Index Scale (Hachinski), Neuropsychiatric Questionnaire (NPI). According to the history and test results by two or more doctors were independently diagnosed, the two determine the results are inconsistent by the third doctor to judge again by the task group members to discuss the case, while the diagnosis and classification of dementia. The diagnosis of dementia refers to the American Psychiatric Diagnostic and Statistical Manual, 4th Edition (DSM-IV), and the diagnosis of senile dementia (AD) is based on the American Society of Neurology, Linguistic Disorders and Stroke-Alzheimer's Disease and Related Diseases (NINCDSADRDA) standards. Assessment of the neuropsychiatric questionnaires (NPI) of patients diagnosed with $\mathrm{AD}$ to evaluate the behavioral and psychological symptoms of dementia (BPSD).

\subsubsection{Statistical methods}

All the data by the unified coding using EpiData3.1 software input, with SAS9.13 statistical analysis of the data.

\section{Results}

\subsection{General Epidemiological data of the study subjects}

A total of 3698 elderly people aged 60 and over were surveyed, with 1916 males and 1782 females. Age: $60 \sim 64$ years old 997 people, 65 to 69 years old 889 people, 70-44 years old 874 people, 75-79 years 517 people, 80 to 84 years old 304 people, 85 years of age 117 people. Education level: illiterate 1249 people, primary school 983 people, junior high school 1207 people, college 259 people. Vascular dementia and mixed dementia were excluded, and a total of 87 cases of senile dementia were diagnosed, the prevalence rate of senile dementia was about $2.4 \%$. Including 35 males and 52 females, the age range of 60 to 90 years, mean age $75.76 \pm 7.22$ years old.

\subsection{Behavioral psychiatric symptoms in senile dementia (AD) patients}

Behavioral and psychological symptoms of dementia (BPSD) are dementia, sensory, mental, or behavioral disorders in dementia patients. $\mathrm{AD}$ patients in the progressive cognitive decline at the same time, often accompanied by loud noisy, too much activity, but also accompanied by delusions, paranoia, etc., is the main source of patient and caregiver heart and body pressure. In order to understand the incidence of common psychiatric symptoms in AD patients, we used the International General Neuropsychiatric Questionnaire (NPI) [2], according to the same standardized survey language, for all diagnosed 87 cases of AD patients within a month of 
neuropsychiatric Symptom to investigate. NPI consists of 12 behavioral domains that cover the most common behavioral disorders in dementia patients. For each behavioral abnormality, the product of the two is the score ( 0 to 12 points) according to the frequency (sub-level 4) and the severity (grade 3), and the sum of the 12 entries is the final of the NPI Score ( $0 \sim 144$ points), the NPI score $>0$ points to determine the NPI abnormalities, indicating that there are mental symptoms (BPSD). In this study, 83.91\% of senile dementia patients had varying degrees of mental and behavioral symptoms in the past month. The most common symptoms were anxiety (54.02\%) and sleep / nocturnal behavior (52.87\%), followed by emotional indifference / Indifference and irritability / emotional instability (both 37.93\%), again for depression / poor mood (33.33\%) and appetite and eating disorders (30.48\%). In addition to inhibition (4.60\%) and emotional high / euphoric (5.75\%), other mental behavior symptoms also have a higher incidence (see Table 1). It is suggested that the incidence of mental disorders in patients with senile dementia is high, should be taken more seriously. In contrast, in the course of the survey, we found that the general population awareness of the disease of senile dementia is very low, in patients with senile dementia, because the disease to take the initiative to visit very little (less than $5 \%$ ), the majority of that is a normal aging phenomenon, by the family or home care services, almost did not get the right treatment, only the individual again serious mental symptoms, only to these symptoms.

Table 1 Alzheimer's disease (AD) Behavioral psychiatric symptoms

\begin{tabular}{cccc}
\hline \multirow{2}{*}{ Mental Behavior Symptom } & $\begin{array}{c}\text { NPI score }>0 \text { number of } \\
\text { cases }\end{array}$ & Incidence (\%) & NPI score ()$(\bar{x} \pm s)$ \\
\hline Delusion & 12 & $13.79 \%$ & $8.00 \pm 1.95$ \\
Illusion & 10 & $11.49 \%$ & $7.20 \pm 1.55$ \\
Agitation/attack & 18 & $20.69 \%$ & $4.56 \pm 1.69$ \\
Depression/bad mood & 29 & $33.33 \%$ & $3.48 \pm 1.55$ \\
Anxiety & 47 & $54.02 \%$ & $3.06 \pm 1.75$ \\
Emotional high/euphoric & 5 & $5.75 \%$ & $2.03 \pm 1.32$ \\
Emotional & 33 & $37.93 \%$ & $5.91 \pm 3.12$ \\
Indifference/Indifference & 4 & $4.60 \%$ & $5.75 \pm 2.87$ \\
$\quad$ Depression & 33 & $37.93 \%$ & $3.58 \pm 1.48$ \\
Irritability/emotional & & & \\
instability & 18 & $20.69 \%$ & $5.28 \pm 2.22$ \\
Abnormal movement & 46 & $52.87 \%$ & $4.41 \pm 2.56$ \\
behavior & 30 & $30.48 \%$ & $4.67 \pm 3.42$ \\
Sleep/nighttime behavior & 79 & $83.91 \%$ & $15.67 \pm 11.56$ \\
Appetite and eating & &
\end{tabular}

\section{Discussion}

In this study, the neurological symptoms of 87 patients with $\mathrm{AD}$ were diagnosed within the last month using the International Symposium on Neuropsychiatric Questionnaire (NPI), according to the same standardized survey language. A total of $83.91 \%$ of patients with senile dementia in the past few months there have been varying degrees of mental behavior symptoms, and foreign reported prevalence of senile dementia $61 \%$ to $88.3 \%$ similar [3-5]. Common and rare forms of 
BPSD are consistent with foreign studies [3,4,7], such as Burns et al. [6] and Lyketsos et al. [3,4] reported anxiety, indifference, depression, agitation / attack lines in dementia The most common ( $24 \%$ to $41 \%$ ), hallucinations, delusions and depressed and emotionally rare (1\% to $10 \%)$. The incidence of depressive symptoms, symptoms of agitation and psychiatric symptoms in psychiatric patients with Alzheimer's disease was as high as 100\%, 93.3\% and 78.3\%, respectively, in the survey of California Alzheimer's behavior questionnaire. This study shows that dementia patients with high incidence of mental behavior, and should be highly valued. In addition, we should strengthen the popularization of knowledge of related diseases, and improve the awareness rate and attendance rate of senile dementia, to prevent the occurrence of senile dementia to give guidance on patients with senile dementia early intervention.

\section{References}

1. Kaufer DL, Miller BL, Itti L, et al . Midline cerebral morphometry distinguishes front otemporal dementia and Alzheimer' s disease . Neurol, 1997; 48 (4): 978.

2. Cummings JL.The neuropsychiatric inventory:assessing psychopathology in dementia patients.Neurology,1997;48 suppl6:s10-s16.

3. Lyketsos CG, Lopez O, Jones B, et al.Prevalence of neuropsychiatric symptoms in dementia: result from the Cardiovascular Health Study.JAMA, 2002; 288:1475-1483.

4. Lyketsos CG,Steinberg M,Tschanz JT,et al.Mental and behavioral disturbances in dementia: findings from the Cache County Study on Memory in Aging. Am J Psychiatry, 2000; 157:708-714.

5. Ikeda M,Fukuhara R,Shigenobu K,et al.Dementia associated mental and behavioral disturbances in elderly people in the community:fin-dings from the first Nakayama study.J Neurol Neurosurg Psychiatry,2004;75:146-148.

6. Burns A,Jacoby R,Leery R.Psychiatric phenomena in Alzheimer's disease.IV : disorders of behavior.Br J Psychiatry,1990;157:86-94.

7. Fuh JL,Liu CK,Mega MS,et al. behavioral disorders and caregivers'reaction in Taiwanese patients with Alzheimer's disease.Int Psychogeriatr,2001;13:121-128.

8. J.H. Sheng, Z.X. Gao, et al., et al.. Investigation of behavior and psychiatric symptoms in Alzheimer's disease patients.Chinese Journal of nervous and mental diseases, 2000; 26:113-116. 